\title{
Alarme noturno e enurese: uma revisão baseada na evidência
}

Joana Pinto Carneiro, ${ }^{1}$ Ana Menezes, ${ }^{1}$ Sofia Correia Pinto ${ }^{2}$

\section{RESUMO}

Introdução: A enurese noturna (EN) é um problema comum em idade pediátrica, atingindo cerca de $15 \%$ das crianças entre os cinco e os seis anos de idade. Pela sua elevada prevalência, várias modalidades terapêuticas têm surgido no sentido de atenuar o seu impacto, muitas vezes estigmatizante para a criança e família. Entre as opções disponíveis para o seu tratamento sobressai o alarme noturno (AN), tratamento comportamental com efeito condicionado a nível do sono.

Objetivo: Rever a evidência científica existente quanto à eficácia do alarme noturno como opção terapêutica no tratamento da enurese noturna.

Metodologia: Foi efetuada uma pesquisa bibliográfica nas principais bases de dados internacionais, utilizando os termos MeSH: Nocturnal enuresis AND Clinical alarms. Foi igualmente consultado o Índex RMP usando os termos de pesquisa (DeCS) "Enurese noturna" e "Alarme". Como critérios de inclusão foram selecionados: meta-análises, revisões sistemáticas, ensaios clínicos aleatorizados, estudos observacionais e orientações clínicas, tendo sido incluídos artigos em língua inglesa e portuguesa, de janeiro de 2005 a janeiro de 2015. Para atribuição do nível de evidência (NE) foi utilizada a escala Oxford Centre for Evidence-Based Medicine 2011.

Resultados: Depois de analisados os 52 artigos resultantes da pesquisa, foram incluídos nove artigos: três revisões sistemáticas, três estudos originais e três normas de orientação clínica. Na maioria da literatura analisada foi identificado benefício acrescido do AN quando comparado com as restantes terapêuticas não farmacológicas. Nos estudos que estabeleceram comparação com a desmopressina, o alarme noturno mostrou resultados equiparáveis, excetuando-se menor risco de recaída com o AN e melhor resposta na EN com capacidade vesical noturna diminuída. Como aspetos menos positivos enumeram-se os resultados mais tardios do $\mathrm{AN}$, a necessidade de colaboração por parte dos pais e o risco de menor adesão ao tratamento. No que respeita às orientações clínicas elaboradas por entidades reconhecidas, as recomendações são concordantes com os resultados dos restantes estudos. Discussão/Conclusão: Considerando os resultados promissores do alarme noturno (Nível de evidência 1, Força de recomendação A), importa que o médico de família considere a sua potencial indicação nos casos de EN resistentes às medidas iniciais, evitando-se a necessidade de intervenção farmacológica e/ou referenciação hospitalar precoce.

Palavras-chave: Enurese noturna; Alarme.

\section{INTRODUÇÃO}

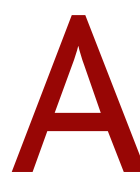

enurese noturna (EN) é um problema comum em idade pediátrica, sendo a queixa urológica mais frequentemente observada ao nível dos cuidados de saúde primários (CSP). ${ }^{1-2}$

Apesar das variáveis étnicas e culturais que tendem a determinar diferenças no significado social da enu-

1. Médica Interna de Medicina Geral e Familiar. USF Garcia de Orta - ACeS Porto Ocidental

2. Médica Interna de Medicina Geral e Familiar. USF S. João do Porto - ACeS Porto Ocidental rese entre as diversas populações, parece existir uma uniformidade na sua prevalência mundial, com atingimento em cerca de $15 \%$ das crianças dos cinco aos seis anos. A partir desta idade, o índice de resolução espontânea é de $15 \%$ ao ano, sendo que a probabilidade de resolução espontânea da enurese é tanto menor quanto maior for a duração da enurese. A EN é também duas a três vezes mais frequente em rapazes do que em

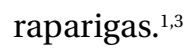

No que respeita aos dados epidemiológicos existem ainda poucos estudos em Portugal. Parte destes foram 
realizados na região Norte e apresentam prevalências distintas, fruto do recurso a diferentes definições de enurese, tamanhos amostrais e idade das populações estudadas, que tornam difícil a comparação entre eles.

Segundo Bandeira e colaboradores, a prevalência de enurese em crianças entre os cinco e os onze anos, no Norte de Portugal, foi de 6,9\%. A procura de ajuda profissional verificou-se apenas em metade dos casos de enurese $(51 \%)$, sendo que apenas em um terço das crianças com enurese foi experimentada alguma forma de tratamento. ${ }^{4}$ Num outro estudo, realizado na região Norte, que incluiu crianças entre os cinco e os seis anos inscritas em 30 unidades de saúde, verificou-se uma prevalência de $16,4 \%$ concordante com a bibliografia relacionada. $^{5}$

Face ao impacto desta problemática na criança e família, têm sido vários os esforços na elaboração de orientações clínicas e uniformização de conceitos no sentido de facilitar a sua abordagem em consulta. Assim, em 2014, a Sociedade Internacional de Continência na Criança (ICCS) entendeu que a enurese noturna poderia ser denominada apenas de enurese, definindo-se como a perda involuntária de urina durante o sono, numa idade em que já é esperado existir controlo vesical ( $>5$ anos). No entanto, por uma questão prática poder-se-á manter o termo corrente de enurese noturna. O uso do termo enurese diurna, por sua vez, foi substituído pela nomenclatura "incontinência diurna", sendo que nictúria corresponde à micção voluntária que ocorre durante a noite. ${ }^{6-8}$ Ainda no que respeita à sua classificação, a EN pode ser definida como primária quando a criança nunca foi continente durante o sono ou secundária quando, após um período mínimo de seis meses de continência, há recorrência das perdas urinárias noturnas. ${ }^{1,6-7}$ Quanto à gravidade da enurese, esta é entendida como ligeira se acontecer menos de duas vezes por semana ou grave se a criança molhar a cama todos os dias da semana. ${ }^{6-7}$

A EN deve ainda ser subclassificada em monossintomática, quando isolada, ou não monossintomática, sempre que existam associados sintomas miccionais diurnos, nomeadamente incontinência urinária diurna, contínua ou intermitente, aumento ou diminuição da frequência das micções, urgência miccional, alterações do jato urinário e/ou infeções urinárias. A enurese noturna monossintomática corresponde à maioria destas crianças (cerca de 80 a 85\% dos casos), sendo esta a tipologia abordada na revisão que se segue..$^{6-7}$

Relativamente à etiologia da EN, esta permanece mal esclarecida, considerando-se provável causa multifatorial. Existe a noção de que se trata de um grupo muito heterogéneo de crianças, em que há sobreposição de inúmeros fatores com intensidades variáveis e daí, também, a grande variabilidade de resposta ao tratamento. ${ }^{9-10}$ Várias teorias têm sido estudadas. A fisiopatologia mais aceite, atualmente, parece assentar no desequilíbrio de três áreas principais: presença de poliúria noturna (resultante da deficiência relativa da hormona antidiurética - vasopressina - durante a noite, com débito urinário superior ou igual a $130 \%$ da capacidade vesical esperada para a idade), capacidade funcional vesical noturna diminuída e dificuldade em despertar a criança enurética. ${ }^{9-10}$

Dos vários trabalhos publicados foi identificado um padrão hereditário, cuja transmissão é autossómica dominante, com penetrância elevada (90\%). Estudos de genética molecular identificaram os cromossomas 12q, 13q e 22 e os respetivos marcadores genéticos relacionados com a enurese noturna, não sendo possível a associação genótipo-fenótipo por haver grande heterogeneidade de locus. Assim, a probabilidade de a criança apresentar enurese se ambos os pais tiveram enurese ou se apenas um teve ou nenhum é de, respetivamente, $77 \%, 44 \%$ e $15 \%$. Esta relação é encontrada na maioria dos estudos, sendo mais frequente história familiar paterna do que materna. ${ }^{11}$

No que respeita ao ambiente psicossocial e familiar sabe-se também que os fatores psicológicos ou psicossomáticos só, raramente, são causa de enurese primária e estão mais vezes implicados na enurese secundária. Desta forma, nas crianças com EN primária, as alterações comportamentais ou psicológicas parecem ser mais uma consequência do que uma causa. ${ }^{12}$

Atendendo à abordagem inicial desta problemática em CSP, é fundamental o esclarecimento da criança e família acerca da fisiopatologia, história natural e evolução da enurese, visando não culpabilizar a criança afetada. A idade em que a enurese é considerada um «problema» varia, dependendo das famílias e do contexto biopsicossocial em que a criança se insere. A EN pode tornar-se significativa para a criança quando interfere com a sua capacidade de socialização (e.g., não 
quer ir dormir à casa dos amigos porque tem vergonha de molhar a cama). ${ }^{1,6-7}$

Numa primeira fase, o médico de família deve privilegiar as recomendações educacionais e comportamentais que passam pela instituição de medidas antienuréticas (insistir na micção ao deitar, no reforço hídrico diurno com restrição nas horas prévias ao deitar); uso de calendário de registo de noites secas e molhadas, com premiação das noites secas (recurso ao «mapa de estrelas»); desaconselhar o uso de fraldas (pelo seu efeito regressivo), favorecendo o uso de proteção de cama e produtos laváveis/descartáveis. A criança deve ser ainda incentivada a assumir responsabilidade, não apenas pelo problema em si, mas também pelo tratamento. Assim, perante cada episódio de perda noturna deve ser a criança a trocar, lavar o pijama e roupas de cama. ${ }^{1,6-7}$

Quando estas opções não são suficientes, o médico de família deve recorrer a outras modalidades terapêuticas que podem, nem sempre, envolver intervenção farmacológica e/ou mesmo referenciação para consulta hospitalar. Um desses exemplos é o alarme noturno (AN), tratamento comportamental com efeito condicionado a nível do sono, cujos resultados têm merecido especial atenção por parte dos profissionais de saúde. Face a este pressuposto, pretende-se com este trabalho rever a evidência existente sobre o uso do AN na EN, estabelecendo linhas orientadoras adequadas à sua abordagem em CSP.

\section{METODOLOGIA}

Foi realizada uma pesquisa bibliográfica nas principais bases de dados internacionais, utilizando os termos MeSH Nocturnal enuresis AND Clinical alarms. Foi igualmente consultado o Índex RMP, usando o termo de pesquisa (DeCS) "Enurese Noturna" e "Alarme". Como critérios de inclusão foram selecionados: meta-análises (MA), revisões sistemáticas (RS), ensaios clínicos aleatorizados (EAC), estudos observacionais e orientações clínicas (NOC), tendo sido incluídos artigos em língua inglesa e portuguesa, de janeiro de 2005 a janeiro de 2015.

A população em estudo incluiu todas as crianças e jovens entre os cinco e os dezoito anos com diagnóstico de EN, cuja intervenção fosse o uso do AN em monoterapia, comparativamente ao uso de placebo, au- sência de qualquer tratamento, outra modalidade terapêutica ou em esquema combinado. O outcome principal baseou-se na redução de noites molhadas/semana e na taxa de resolução da enurese (14 noites secas consecutivas). Foram excluídos os estudos que não cumprissem os critérios de inclusão, por divergirem do objetivo do trabalho, por serem artigos repetidos ou estarem incluídos nas MA ou nas RS.

Para atribuição do nível de evidência (NE) foi utilizada a escala Oxford Centre for Evidence-Based Medicine 2011.

\section{RESULTADOS}

A pesquisa efetuada resultou na identificação de 52 artigos, tendo sido excluídos 43. Dos contemplados na revisão consideraram-se três revisões sistemáticas, três estudos originais e três normas de orientação clínica, perfazendo um total de 6.393 crianças e jovens estudados.

A descrição dos artigos encontra-se resumida por categorias nos Quadros I, II e III.

A revisão sistemática da Cochrane, de Glazener e colaboradores, publicada em 2005, teve como objetivo principal avaliar a eficácia do AN na redução da taxa de enurese (definida como 14 noites secas consecutivas) em comparação com nenhum tratamento, abordagem comportamental simples (que contempla treino de retenção vesical, mapa de noites secas com recompensa, despertar noturno, micção noturna programada), desmopressina e outras intervenções (uso de antidepressivos tricíclicos, outros fármacos, psicoterapia, dieta, alarme com recurso a choques elétricos). Os outcomes secundários consistiram na avaliação da redução do número médio de noites molhadas por semana, na taxa de recidiva após sucesso inicial, no número médio de noites molhadas/semana após cessar o tratamento e na presença de eventos adversos. Nesta revisão foram incluídos 56 ensaios clínicos aleatorizados que contemplaram um total de 3.257 crianças, das quais 2.345 usaram AN, entre os cinco e os 16 anos. ${ }^{13}$

Os resultados mostraram que $2 / 3$ das crianças atingiram 14 noites secas consecutivas com o uso de AN $v s$. $4 \%$ do grupo controlo sem tratamento (risco relativo [RR] para falha do tratamento 0,38 ; intervalo de confiança a 95\% [IC]: 0,33-0,45). No que respeita à abordagem comportamental simples, não houve diferenças 


\section{QUADRO I. Revisões sistemáticas}

\begin{tabular}{|c|c|c|}
\hline \multirow{2}{*}{$\begin{array}{l}\text { Autor/Ano } \\
\text { Glazener, et al. } \\
(2005)\end{array}$} & & NE \\
\hline & $\begin{array}{l}\text { Intervenções: AN vs. nenhum tratamento; AN vs. abordagem comportamental simples*; AN vs. } \\
\text { desmopressina; AN vs. outras intervenções. } \\
\text { Outcome: Redução da taxa de enurese** } \\
56 \text { ECA }^{* * *} \text { ( } n=3.257 \text { crianças, } 2.345 \text { usaram AN) } \\
\text { Resultados: } \\
\text { - Desmopressina apresentou efeito mais imediato, embora o AN tenha provas de maior sucesso a } \\
\quad \text { longo prazo [ } 4 / 22 \text { ( } 18 \% \text { ) vs. } 16 / 24(67 \%), \text { RR de } 0,27 ; \text { IC de } 95 \%: 0,11-0,69 \text { ]. } \\
\text { - AN foi mais eficaz que os tricíclicos durante o tratamento (RR } 0,73 ; \text { IC de } 95 \%: 0,61-0,88 \text { ). } \\
\text { - Não houve diferenças significativas entre o AN e outras intervenções simples comportamentais. }\end{array}$ & 1 \\
\hline $\begin{array}{l}\text { Caldwell, et al. } \\
\text { (2013) }\end{array}$ & $\begin{array}{l}\text { Intervenções: Métodos comportamentais simples (MCS)*** vs. nenhum tratamento; MCS isolada vs. } \\
\text { outro método comportamental (outro simples, AN ou combinado); MCS vs. tratamento farmacológico } \\
\text { (em monoterapia ou em combinação). } \\
\text { Outcome: Redução da taxa de enurese**; redução do número de noites molhadas/semana. } \\
16 \text { ECA ( } n=1.643 \text { crianças, } 865 \text { c/MCS) } \\
\text { Resultados: } \\
\text { - MCS detém eficácia inferior ao AN, tendo por base a redução de noites molhadas/semana (RR } 2,25 \text {; } \\
\text { IC de } 95 \%: 0,30-4,20 \text { ). } \\
\text { - AN foi o método mais eficaz em atingir } 14 \text { noites secas consecutivas (RR } 2,73 \text {; IC de } 95 \%: 1,75-4,26 \text { ). }\end{array}$ & 1 \\
\hline $\begin{array}{l}\text { Kiddoo, et al. } \\
\text { (2015) }\end{array}$ & $\begin{array}{l}\text { Intervenções: Dry bed training***** vs. nenhum tratamento; Dry bed training vs. AN; Dry bed training e } \\
\text { AN vs. nenhum tratamento; AN vs. nenhum tratamento; hipnoterapia vs. AN; acupuntura vs. acupuntura } \\
\text { simulada. } \\
\text { Outcome: número de noites secas ininterruptas } \\
5 \text { ECA ( }=969 \text { crianças) } \\
\text { Resultados: } \\
\text { - Maior número de noites secas consecutivas com AN. } \\
\text { - Uso combinado do AN e Dry bed training demonstrou maior eficácia comparativamente com a } \\
\text { acupuntura, hipnoterapia e Dry bed training isolado. }\end{array}$ & 1 \\
\hline
\end{tabular}

significativas entre esta e o AN. A desmopressina, por sua vez, teve efeito mais imediato na redução das noites molhadas, embora o AN tivesse provas de maior sucesso a longo prazo [4/22 (18\%) vs. 16/24 (67\%), RR de 0,27; IC de 95\%: 0,11-0,69]. Em relação aos outros tipos de intervenções, o AN foi mais eficaz, particularmente em comparação com os antidepressivos tricíclicos (RR 0,73; IC de 95\%: 0,61-0,88). Em contrapartida, os autores acrescentam que o preço do tratamento com tricíclicos ou drogas afins é consideravelmente mais acessível do que com o AN ou a desmopressina.

Ainda relativamente aos resultados desta RS, identificou-se maior eficácia do AN na enurese frequente (mais de duas vezes por semana) e nas crianças mais velhas ( $>7$ ou 8 anos de idade). Este dispositivo poderá ser usado, no entanto, em crianças mais novas, dependendo da capacidade da criança, motivação e compreensão do alarme. Quanto ao benefício em associar o AN à desmopressina, os resultados não foram elucidativos.

A utilização de um alarme imediato que acorda a criança (em comparação com um alarme que acorda apenas os pais) mostrou melhores resultados. Num dos ensaios, as crianças preferiram um AN junto ao corpo do que junto à almofada, bem como um alarme sonoro versus do tipo vibratório mas, de um modo geral, não houve evidência de superioridade de um em relação a outro. 


\begin{tabular}{|c|c|c|}
\hline Autor/Ano & & NE \\
\hline $\begin{array}{l}\text { Kwak, et al. } \\
\text { (2010) }\end{array}$ & $\begin{array}{l}\text { ECA ( } 127 \text { crianças } \geq 5 \text { anos com EN) } \\
\text { Intervenção: Desmopressina vs. AN } \\
\text { Outcome: Redução da taxa de enurese**; recaída (>1 episódio de enurese/semana) } \\
\text { Resultados: } \\
\text { - Eficácia na redução da taxa de enurese comparável entre desmopressina e AN }(77,8 \% \text { e } 82 \% \text {, } \\
\text { respetivamente) } \\
\text { - Recidiva mais frequente após o tratamento com desmopressina ( } 50 \% \text { vs. } 12 \% \text { (AN) }(p=0,005) \text {. }\end{array}$ & 2 \\
\hline $\begin{array}{l}\text { Evans, et al. } \\
\text { (2011) }\end{array}$ & $\begin{array}{l}\text { ECA [ } 251 \text { crianças } \geq \text { 5anos com EN severa (cerca de } 5,5-5,6 \text { noites molhadas/semana)] } \\
\text { Intervenção: Desmopressina vs. AN } \\
\text { Outcome: redução do número de noites molhadas/semana } \\
\text { Resultados: Não houve diferença entre desmopressina e AN durante o tratamento para atingir noites } \\
\text { secas ( } 37,5 \% \text { vs. } 32,2 \%) \text {. A adesão ao tratamento com AN durante o estudo foi menor em comparação } \\
\text { com a desmopressina ( } 50-78 \% \text { vs. } 95-98 \% \text { ). }\end{array}$ & 2 \\
\hline $\begin{array}{l}\text { Önol, et al. } \\
(2014)\end{array}$ & $\begin{array}{l}\text { ECA ( } n=146 \text { crianças) com EN monossintomática primária sem resposta ou com resposta parcial } \\
\text { ao tratamento inicial } \\
\text { Intervenções: Tratamento aleatório com AN e desmopressina, durante } 6 \text { meses; eficácia a longo termo } \\
\text { entre AN e a desmopressina liofilizada. } \\
\text { Outcome: Compliance ao tratamento de forma mensal, recorrendo ao diário de noites molhadas. Taxas } \\
\text { de sucesso: redução de >90\% noites molhadas por mês ao fim dos } 6 \text { meses e aos } 12 \text { meses. } \\
\text { Resultados: } \\
\text { - Maior taxa de abandono com o AN ( } 30,7 \% \text { vs. } 5,2 \%) \text {. A taxa de sucesso aos } 6 \text { meses foi } 76,8 \% \text { e } \\
\quad 61,8 \% \text { nas crianças com desmopressina e AN, respetivamente. } \\
\text { - Aos } 12 \text { meses, } 77,8 \% \text { das crianças que tinham sido medicadas com desmopressina e } 75 \% \text { das que } \\
\text { usaram AN, não apresentaram recidiva de EN. }\end{array}$ & 2 \\
\hline
\end{tabular}

Entre as desvantagens do uso do AN, os autores referem o seu efeito mais tardio em relação à desmopressina (duração mínima do tratamento é de três meses) e a necessidade de colaboração por parte dos familiares, com consequente maior abandono da terapêutica. No que respeita aos eventos adversos, apenas um estudo declarou explicitamente não haver efeitos secundários. Os restantes relataram avaria do alarme, falsos positivos, suscetível de assustar a criança ou familiares durante a noite, potenciando episódios de conflito familiar. Em dois dos ensaios, o não cumprimento ou desistência do uso de AN foi atribuído à dificuldade em utilizar e compreender o funcionamento do equipamento.

Entre os estudos incluídos, o método de ocultação de grupos foi de boa qualidade metodológica em apenas oito dos ensaios. De referir também que sete ensaios foram do tipo quasi-experimental. Apenas 26 dos ensaios incluídos avaliaram os resultados a longo prazo após o tratamento concluído, situação considerada limitativa, uma vez que a eficácia continuada é o objetivo principal do tratamento.

Na revisão sistemática da Cochrane, de Caldwell e colaboradores, publicada em 2013, foi avaliada a eficácia dos métodos comportamentais simples (MCS), que consistem em comportamentos ou ações que podem ser alcançados pela criança sem grande esforço. Tais métodos incluíram a realização de treino de retenção vesical, recurso ao mapa de noites secas, sistema de recompensa de noites secas e despertar noturno programado. Os MCS foram avaliados em comparação com nenhum tratamento, outro método comportamental 
QUADRO III. Orientações clínicas

\begin{tabular}{|c|c|c|}
\hline & & FR \\
\hline $\begin{array}{l}\text { National Institute for } \\
\text { Health and Care } \\
\text { Excellence }^{21} \\
\text { NICE Guidelines } \\
\text { October } 2010\end{array}$ & $\begin{array}{l}\text { "O AN deve ser usado como tratamento de primeira linha nas crianças com EN sem resposta } \\
\text { eficaz com as medidas educacionais". "(...) está particularmente indicado se a criança } \\
\text { apresentar baixa capacidade vesical e familiares que colaborem no processo terapêutico, } \\
\text { devendo ser complementada com outros métodos comportamentais simples." }\end{array}$ & $A$ \\
\hline $\begin{array}{l}\text { International Children's } \\
\text { Continence Society }{ }^{19} \\
\text { J Urol. February } 2010\end{array}$ & $\begin{array}{l}\text { "A abordagem primária da EN deve incluir o treino vesical, o AN e/ou a desmopressina". "O AN } \\
\text { deve ser considerado em todas as crianças, especialmente se os pais estiverem motivados". "A } \\
\text { desmopressina é a alternativa mais adequada na EN com poliúria noturna". }\end{array}$ & $A$ \\
\hline $\begin{array}{l}\text { Practical consensus } \\
\text { guidelines for the } \\
\text { management of } \\
\text { enuresis }{ }^{20} \\
\text { Eur J Pediatr. } 2012\end{array}$ & $\begin{array}{l}\text { "O AN bem como a desmopressina são intervenções eficazes para a EN em crianças que } \\
\text { apresentem resposta insuficiente com as medidas iniciais. A escolha da terapêutica nestes } \\
\text { casos depende do tempo de resposta desejada, do empenho da família, presença de poliúria } \\
\text { noturna e capacidade vesical." }\end{array}$ & $A$ \\
\hline
\end{tabular}

(outro simples, AN ou MCS combinado) e com tratamento farmacológico (através do recurso à desmopressina, oxibutinina, imipramina ou amitriptilina). Os outcomes primários consistiram na redução da taxa de enurese (definida como 14 noites secas consecutivas) e na redução do número de noites molhadas por semana, após término do tratamento. Como outcomes secundários consideraram-se: o número de noites molhadas por semana a longo prazo após o tratamento estar concluído, taxa de recaída e presença de eventos adversos. Foram incluídos 16 ensaios clínicos aleatorizados para um total de 1.643 crianças, 865 das quais com MCS. ${ }^{14}$

Dos resultados obtidos, os MCS parecem ter eficácia inferior ao AN, tendo por base a redução de noites molhadas/semana (RR 2,25; IC de 95\%: 0,30-4,20). A única comparação, em que se verificou diferença estatisticamente significativa, foi entre o AN e o treino de retenção vesical. AN foi também o método mais eficaz em atingir 14 noites secas consecutivas (RR 2,73; IC de 95\%: 1,75-4,26). Os autores deste estudo referem, no entanto, que a qualidade metodológica dos 16 estudos incluídos foi baixa, com amostras pequenas na maioria dos estudos. De acrescentar também que apenas 10 dos 16 estudos deram informações sobre os resultados de acompanhamento após a intervenção ter sido concluída. Outro potencial viés está relacionado com a falta de informação sobre os participantes, nomeadamente se houve inclusão de crianças com causas orgânicas de enurese ou incontinência urinária diurna (que podem responder de forma diferente às intervenções).

Relativamente à revisão sistemática de Kiddoo, 2015, foram incluídos cinco ECA (n=969 crianças). As intervenções contempladas abrangeram o uso do Dry bed training (programa intensivo que combina mapa de noites secas com recompensa, medidas antienuréticas como micção ao deitar, despertar noturno para esvaziar a bexiga, reforço hídrico diurno e restrição nas horas prévias ao deitar) vs. nenhum tratamento; Dry bed training vs. AN; Dry bed training e AN vs. nenhum tratamento, AN $v s$. nenhum tratamento, hipnoterapia $v s$. AN, acupuntura $v$ s. acupuntura simulada. O outcome primário baseou-se na capacidade de atingir o maior número de noites secas ininterruptas. Os outcomes secundários incluíram: número médio de noites molhadas por semana, taxa de recidiva após o sucesso inicial, número médio de noites molhadas após o fim de tratamento e presença de efeitos adversos. ${ }^{15}$

Dos resultados apresentados verificou-se maior número de noites secas consecutivas com o AN. O uso combinado do AN e Dry bed training demonstrou também maior eficácia, comparativamente com a acupuntura, hipnoterapia e Drybed training isolados. Neste estudo, os autores consideram que o AN representa 
um dos tratamentos mais eficazes a longo prazo e com poucos efeitos secundários.

O ECA, de Kwak e colaboradores, publicado em 2010, foi desenhado com o objetivo de avaliar a eficácia do AN em crianças com enurese resistente ao tratamento farmacológico (pharmacotherapy-resistant nocturnal enuresis - PRNE). As crianças foram divididas em três grupos: no grupo 1 foram incluídas crianças com resposta parcial ou ausência de resposta à desmopressina; no grupo 2 , aquelas que não obtiveram resposta satisfatória com desmopressina mais oxibutinina; no grupo 3, crianças que apresentaram recidiva de EN após sucesso prévio com farmacoterapia. Neste estudo foram incluídas 127 crianças ( 100 no final) com EN e idade igual ou superior a cinco anos. ${ }^{16}$

No geral, $53,7 \%, 40 \%$ e $52,4 \%$ das crianças dos grupos 1, 2, e 3 apresentaram resposta completa (RC) com AN, respetivamente. Estas diferenças não foram estatisticamente significativas $(p=0,798)$. Nos grupos 1 e 2 , $54,4 \%(31 / 57)$ dos que apresentavam benefício parcial prévio com farmacoterapia e $36,4 \%(8 / 22)$ daqueles sem resposta primária tiveram RC com AN.

Em termos de capacidade da bexiga, havia 60 crianças com capacidade vesical noturna diminuída e 40 com uma capacidade de bexiga normal. Destas, 34 $(56,7 \%)$ e 16 (40\%), respetivamente, apresentaram RC após o uso de AN. No entanto, esta diferença não alcançou significância estatística ( $p=0,247)$.

Do total de crianças com RC, $60,7 \%, 88,9 \%$ e $54,5 \%$ dos grupos 1, 2, e 3 não apresentaram recaída seis meses após a interrupção do tratamento com o AN, respetivamente. Este estudo permitiu determinar a utilidade do AN como estratégia terapêutica eficaz para crianças com PRNE.

O ECA de Evans e colaboradores, de 2011, por sua vez, pretendeu avaliar a eficácia a longo prazo da desmopressina em comparação com o AN. Este estudo contemplou apenas crianças $\geq 5$ anos de idade com EN grave (média 5,5-5,6 noites molhadas/semana), perfazendo um total de 251 crianças divididas em dois grupos: um sob terapêutica com desmopressina $(0,2-0,4 \mathrm{mg}$ por dia) e outro com AN. A eficácia foi avaliada pela redução percentual no número médio de noites molhadas/semana ao longo de 12 meses (seis meses de tratamento + seis meses de follow up) pelo número de crianças que atingiram continência, pela duração média do sono e adesão à terapêutica. ${ }^{17}$

Relativamente aos resultados obtidos, cerca de $32 \%$ das crianças que usavam o AN desistiram contra cerca de $7 \%$ das que começaram com desmopressina. Numa análise global foi encontrada uma proporção similar na redução das noites molhadas/semana em ambos os grupos (desmopressina: $37,5 \%$ vs. AN: $32,2 \%$ ), bem como na capacidade em atingir continência total (desmopressina: $32 \% v s$. AN: $37 \%$ ). A adesão foi maior com desmopressina: 95-98\% de pacientes tomaram mais de $75 \%$ dos comprimidos $v$ s. os $50-78 \%$ que usaram AN mais de $75 \%$ das noites. A duração média do sono aumentou em cerca de $1,02 \mathrm{~h}$ no final do tratamento com desmopressina (IC 95\%: 0,045, 1,99). O AN apresentou, assim, resultados equiparáveis à desmopressina em atingir as noites secas, apesar da menor adesão ao tratamento.

O ECA de Önol e colaboradores, publicado em 2014, pretendeu, de igual forma, avaliar a eficácia a longo termo entre AN e a desmopressina liofilizada em crianças com EN monossintomática, procurando determinar, em simultâneo, quais os fatores preditores no sucesso da terapêutica. ${ }^{18}$

Foram incluídas 146 crianças com EN monossintomática primária tratadas aleatoriamente com $\mathrm{AN}$ e desmopressina durante seis meses. Durante este período foi avaliada a compliance ao tratamento de forma mensal, recorrendo ao diário de noites molhadas. Foram calculadas as taxas de sucesso (redução mais de $90 \%$ noites molhadas por mês) ao fim dos seis meses e aos 12 meses (seis meses após término da terapêutica).

Assim como sucedeu no ECA de Evans, registou-se maior taxa de abandono com o AN (30,7\% vs. 5,2\%). A taxa de sucesso aos seis meses foi $76,8 \%$ e $61,8 \%$ nas crianças com desmopressina e AN, respetivamente. Aos 12 meses, $77,8 \%$ das crianças que tinham sido medicadas com desmopressina e $75 \%$ das que usaram AN não apresentaram recidiva de EN. No entanto, ajustando a taxa de sucesso com a intenção de tratar, esta foi mais alta para a desmopressina $(68,8 \% v s .46,2 \%)$. Da análise das multivariáveis verificou-se que a terapia de grupo, a severidade da EN ( $>5$ noites molhadas/semana) e a avaliação mensal pelos médicos assistentes foram fatores preditores independentes de cura aos seis meses de tratamento.

Segundo as orientações da International Children's 
Continence Society (ICCS), publicada em 2010, a abordagem primária da EN deve incluir o treino vesical, o AN e/ou a desmopressina. O AN deve ser considerado em todas as crianças, especialmente se os pais estiverem motivados. A desmopressina, por outro lado, é a alternativa mais adequada na EN com poliúria noturna (força de recomendação A). ${ }^{19}$

Relativamente aos consensos publicados em 2012 pela Sociedade Europeia de Nefrologia Pediátrica e pela Academia Americana de Pediatras, o AN bem como a desmopressina são intervenções eficazes para a EN em crianças que apresentem resposta insuficiente com as medidas iniciais. A escolha da terapêutica, nestes casos, depende do tempo de resposta desejada, do empenho da família, da presença de poliúria noturna e da capacidade vesical noturna (força de recomendação A). ${ }^{20}$

Com base nas guidelines do National Institute for Health and Care Excellence (NICE), de 2010 e reformuladas em 2015, recomenda-se o AN como tratamento de primeira linha nas crianças com EN sem resposta eficaz às medidas iniciais educacionais. Está indicado, em particular, se a criança apresentar baixa capacidade vesical noturna e familiares que colaborem no processo terapêutico, devendo ser complementada com outros métodos comportamentais simples (força de recomendação A). ${ }^{21}$

As três NOCs recomendam, assim, com uma força de recomendação A, que o AN deve estar entre as opções de primeira linha no tratamento da EN, sempre que as medidas iniciais não forem suficientes para reverter o quadro. ${ }^{19-21}$

\section{CONCLUSÃO}

$\mathrm{Na}$ maioria da literatura analisada foi identificado benefício acrescido do AN quando comparado com as restantes terapêuticas não farmacológicas. Nos estudos que estabeleceram comparação com a desmopressina, o AN mostrou resultados equiparáveis, excetuando-se menor risco de recaída com o AN e melhor resposta na EN com capacidade vesical noturna diminuída. Como aspetos menos positivos enumeram-se os resultados mais tardios do AN, a necessidade de colaboração por parte dos familiares e o risco de menor adesão ao tratamento. Ressalva-se ainda a resposta mais eficaz da desmopressina na EN com poliúria noturna.
No que respeita às orientações clínicas elaboradas por entidades reconhecidas e acima descritas, as recomendações são concordantes com os resultados do estudo.

Considerando os resultados promissores do AN (Nível de evidência 1, Força de recomendação A), importa que o médico de família considere a sua potencial indicação nos casos de EN resistentes às medidas iniciais, evitando-se a necessidade de intervenção farmacológica e/ou referenciação hospitalar.

\section{REFERÊNCIAS BIBLIOGRÁFICAS}

1. Gonzalez Jr ET, Tu ND. Approach to the child with nocturnal enuresis. UpToDate. 2009;17(1).

2. Reis A, Coelho P. Enurese nocturna: orientação pelos cuidados de saúde primários [Nocturnal enuresis: management in primary health care]. Rev Port Clin Geral. 2007;23(3):279-88. Portuguese

3. Hay WW, Levin MJ, Sondheimer JM, Deterding RR. Current diagnosis \& treatment in pediatrics. 18th ed. McGraw Hill; 2007. p. 204-6. ISBN 9780071463003

4. Bandeira A, Barreira JL, Matos P. Prevalência da Enurese Noturna em crianças em idade escolar na Zona Norte de Portugal [Prevalence of nocturnal enuresis in children of elementary school age]. Nascer Crescer. 2007; XVI(2):65-9. Portuguese

5. Gomes CA, Henriques S, Tavares A, Fonseca C. Enurese em crianças portuguesas: prevalência e relação com hábitos de sono e pesadelos [Enuresis in Portuguese children: a study of its prevalence and association with sleep habits and nightmares]. Rev Port Med Geral Fam. 2012;28(5):338-42. Portuguese

6. Austin PF, Bauer SB, Bower W, Chase J, Franco I, Hoebeke P, et al. The standardization of terminology of lower urinary tract function in children and adolescents: update report from the Standardization Committee of the International Children's Continence Society. J Urol. 2014; 191(6):1863-5.e13.

7. Nevéus T, von Gontard A, Hoebeke P, Hjälmås K, Bauer S, Bower W, et al. The standardization of terminology of lower urinary tract function in children and adolescents: report from the Standardisation Committee of the International Children's Continence Society. J Urol. 2006;176 (1):314-24

8. American Psychiatric Association. Enuresis. In: Diagnostic and statistical manual of mental disorders DSM-IV. Washington: APA; 2000.

9. Caldwell PH, Deshpande AV, Von Gontard A. Management of nocturnal enuresis. BMJ. 2013;347:f6259.

10. Butler RJ, Holland P. The three systems: a conceptual way of understanding nocturnal enuresis. Scand J Urol Nephrol. 2000;34(4):270-7.

11. Von Gontard A, Schaumburg H, Hollmann E, Eiberg H, Rittig S. The genetics of enuresis: a review. J Urol. 2001;166(6):2438-43.

12. Costa T. Enurese nocturna: fisiopatologia e tratamento. Rev Hosp Crianças Maria Pia. 2006;XV(3):S174-9.

13. Glazener CM, Evans JH, Peto RE. Alarm interventions for nocturnal enuresis in children. Cochrane Database Syst Rev. 2005;(2):CD002911.pub2.

14. Caldwell PH, Nankivell G, Sureshkumar P. Simple behavioural interven- 
tions for nocturnal enuresis in children. Cochrane Database Syst Rev. 2013; (7):CD003637.

15. Kiddoo D. Nocturnal enuresis: non-pharmacological treatments. BMJ Clin Evid. 2015 Jan 13;2015. pii:0305.

16. Kwak KW, Lee YS, Park KH, Baek M. Efficacy of desmopressin and enuresis alarm as first and second line treatment for primary monosymptomatic nocturnal enuresis: prospective randomized crossover study. J Urol. 2010;184(6):2521-6.

17. Evans J, Malmsten B, Maddocks A, Popli HS, Lottmann H. Randomized comparison of long-term desmopressin and alarm treatment for bedwetting. J Pediatr Urol. 2011;7(1):21-9.

18. Önol F, Guzel R, Tahra A, Kaya C, Boylu U. Comparison of long-term efficacy of desmopressin lyophilisate and enuretic alarm for monosymptomatic enuresis and assessment of predictive factors for success: a randomized prospective trial. J Urol. 2015;193(2):655-61.

19. Neveus T, Eggert P, Evans J, Macedo A, Rittig S, Tekgül S, et al. Evaluation of and treatment for monosymptomatic enuresis: a standardization document from the International Children's Continence Society. J Urol. 2010;183(2):441-7.
20. Vande Walle J, Rittig S, Bauer S, Eggert P, Marschall-Kehrel D, Tekgul S. Practical consensus guidelines for the management of enuresis. Eur J Pediatr. 2012;171(6):971-83.

21. National Clinical Guideline Centre. Nocturnal enuresis: the management of bedwetting in children and young people [Internet]. London: NICE; 2010. Available from: https://www.nice.org.uk/guidance/cg111/ evidence/cg111-nocturnal-enuresis-the-management-of-bedwettingin-children-and-young-people-full-guideline3

\section{CONFLITO DE INTERESSES}

As autoras declaram não ter conflitos de interesse.

\section{ENDEREÇO PARA CORRESPONDÊNCIA \\ Joana Pinto Carneiro \\ USF Garcia de Orta - ACeS Porto Ocidental \\ E-mail: medjoanacarneiro@gmail.com}

Recebido em 16-02-2016

Aceite para publicação em 01-05-2017

\section{ABSTRACT}

\section{NOCTURNAL ALARM THERAPY AND ENURESIS: AN EVIDENCE-BASED REVIEW}

Introduction: Nocturnal enuresis is a common problem, affecting about $15 \%$ of children between the ages of five and six years. Different treatments have been studied in order to diminish the potentially stigmatizing impact of NE, both for the child and the family. These include nocturnal alarms and behavior modification affecting sleep.

Aim: The aim of this review was to assess the evidence for the effectiveness of nocturnal alarms in the treatment of nocturnal enuresis.

Methods: A bibliographic review of international databases was conducted, using the MeSH terms 'Nocturnal enuresis' AND 'Clinical alarms'. We also consulted the Índex RMP database using the search terms Enurese noturna and Alarme. We selected meta-analyses, systematic reviews, randomized controlled trials, observational studies, and clinical guidelines written in Portuguese and English that were published between January 2005 and January 2015. The Oxford Centre for Evidence-Based Medicine criteria were used to establish the Level of Evidence (LE).

Results: From a total of 52 articles found, nine met the inclusion criteria. These included three systematic reviews, three clinical trials, and three guidelines. Most studies found that NA is more beneficial than other non-pharmacologic options. When compared to desmopressin, the use of NA has shown equivalent effectiveness. Its use is associated with less risk of relapse and a better response when NE occurs due to diminished nocturnal bladder capacity. However, beneficial results take longer to appear with NA, parental collaboration is needed, and there is a risk of decreased adherence to this treatment. Clinical guidelines agree with the results of published trials.

Discussion: Given the potential benefits of nocturnal alarms in the treatment of nocturnal enuresis (Level of Evidence 1, Strength of Recommendation A), the family doctor should consider this treatment for NE resistant to initial measures, thus avoiding the need for pharmacologic intervention or premature referral.

Keywords: Nocturnal enuresis; Clinical alarms. 